\title{
Glossines d'Afrique centrale III. Espèces rares du groupe Palpalis
}

\author{
par L. MAILLOT (suite)*
}

Glossina newsteadi Austen, Glossina caliginea Austen et Glossina pallicera Bigot sont des espèces rares ou très rares en Afrique centrale. Toutes trois sont des espèces de forêt, mais $G$. newsteadi la plus fréquente se trouve en général aux limites du massif forestier équatorial, elle existe au Mayumbe en Angola (7), au Cabinda (31), au Congo-Léopoldville au sud de la forêt équatoriale (2), elle a été déterminée au Gabon (districts de Kango et de Mayumba), au Congo : dans le Mayumbe (district de M'Vouti), dans la Sangha-Likouala (districts d'Ewo, de Kellé, d'Ouesso et de Souanké), dans la Likouala (district de Dongou) ; en R.C.A. : dans la HauteSangha (district de Nola); G. caliginea et G. pallicera sont beaucoup plus rares, on ne les trouve qu'au nord du Gabon ef en Haute-Sangha (11).

Des trois espèces $G$. newsteadi est la moins hygrophile (11). En Afrique Centrale, G. caliginea et $G$. pallicera semblent être, comme $G$. medicorum, à la limite extrême méridionale de leur zone d'expansion. C'est sans doute la raison pour laquelle $G$. pallicera n'y a été que si rarement identifiée (ROUBAUD - 14-. GALLIARD $30-$ ). On pourrait par ailleurs considérer que c'esł une espèce en voie de disparition et il me paraît peu probable qu'elle ait pu très souvent être confondue avec $G$. newsteadi ; par contre cette dernière espèce $a$, en certains cas, été cataloguée comme une forme aberrante de G. pollicero (cf. HEGH et MACHADO - 7 -).

Dans les régions où ces trois espèces ont été rencontrées le régime pluviométrique est le suivant :

pour G. pallicera en moyenne de 1500 à $1750 \mathrm{~mm}$ de pluie par an avec une saison sèche de 2 à 3 mois ;

* Voir Rev. Elev. Méd. vet. Poys trop., 1961. 14 (4) :439-43

Rev. Elev. Méd. vét. Pays trop., 1962, 15, n 1.

Reçu pour publication; Avril 1962. pour $G$. caliginea en moyenne de 1500 d̀ $2500 \mathrm{~mm}$ de pluie par an avec pas de saison sèche ou une saison sèche d'une, durée maximum de 2 à 3 mois ;

pour $G$. newsteadi en moyenne de $1400 \mathrm{~mm}$ d̀ $2500 \mathrm{~mm}$ de pluie par an avec pas de saison sèche ou une saison sèche au maximum de 4 mois.

\section{DÉTERMINATION}

Ces trois espèces quelquefois signalées dans la même région sont très voisines par certains caractères de la morphologie extérieure, taille, couleur (39). G. palliccra cst ccpendant facilement différenciée par la forme: générale du $3^{e}$ segment de l'antenne et la longueur des poils du liseré antennaire.

Ce sont en outre les examens des genitalia qui permettront la différenciation de ces espèces, les principaux caractères sont les suivants : pour $G$. caliginea la forme des cerques chez le mâle (fig. I), la forme des plaques anales (en aile de papillon) chez la femelle (fig. III) ; pour G. pallicera et pour $G$. newsteadi chez le mâle la forme des gonopodes est distincte (fig. IV et $V \mid$ ), chez la femelle pour ces deux espèces les plaques anales les différencient bien des femelles de gl. palpalis et de G. fuscipes ; ces plaques (fig. $V$ et VII) sont d'aspects piriformes mais paraissent assez souvent plus courtes ef plus sphériques chez $G$. newsteadi : dans cette dernière espèce les plaques dorsales sont peu élevées et présentent d'une façon constante une encoche ou espace clair à l'angle inférointerne.

La comparaison de l'ensemble de ces caractères justifie la nouvelle classification de MACHADO (7), qui ramène $G$. newsteodi au rang de sous-espèce : $G$. pallicera newsteadi, $G$. pallicera prenant l'appellation de $G$. pallicera pollicera. 


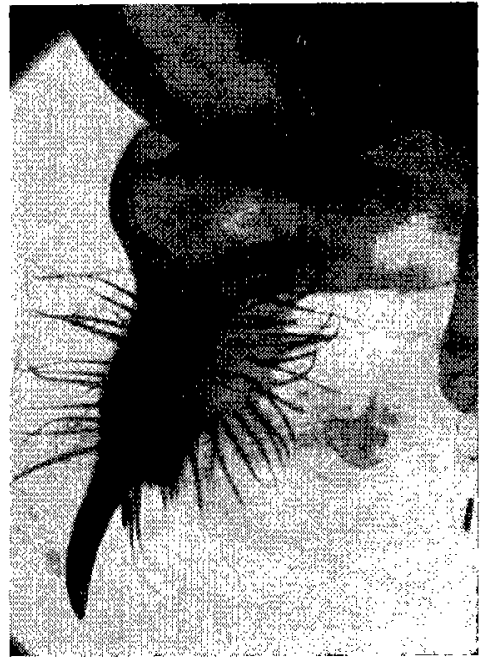

Fig. I. - 'G. coliginea, mâle, cerques, Kango, fleuve Como, Gabon.

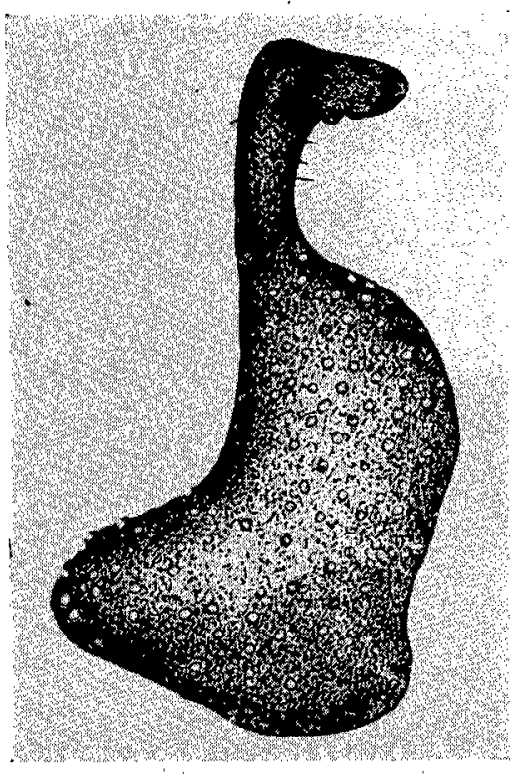

Fig. IV. - G. newsteadi, mâle gonopode, d'après Patton.

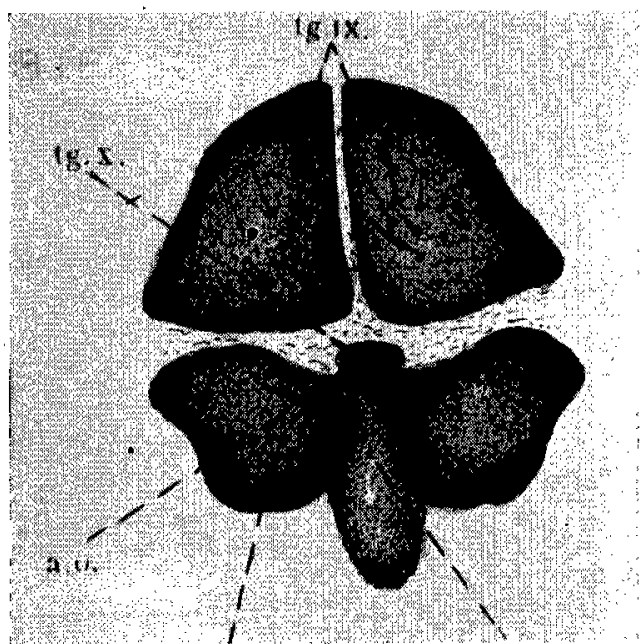

Fig. III. - G. caliginen, femelle, plaques génitales d'après Patton.

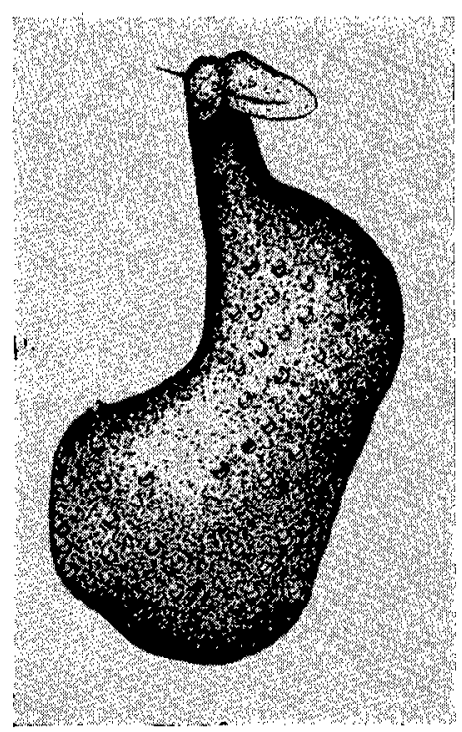

Fig. VI. - G. pallicera, mâle, gonopode,
d'après Patton. 


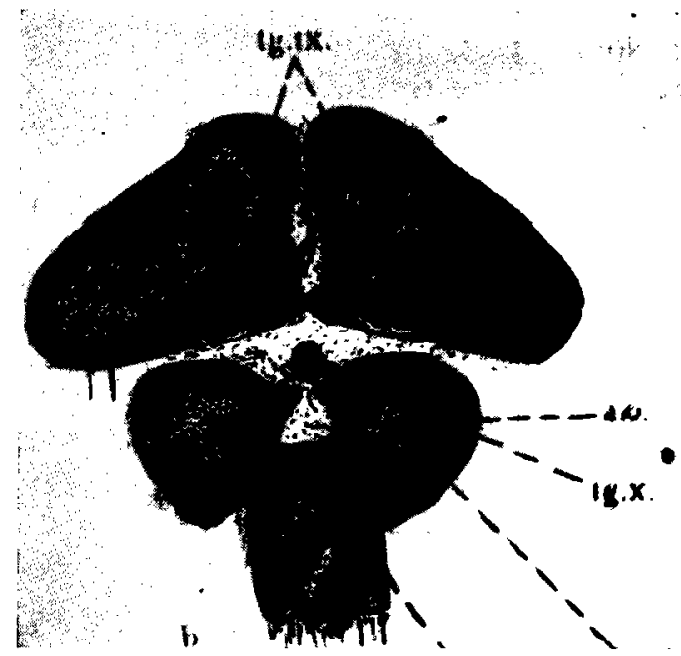

Fig. V. - G. newsteadi, femelle, plaques génitales, d'après Patton.

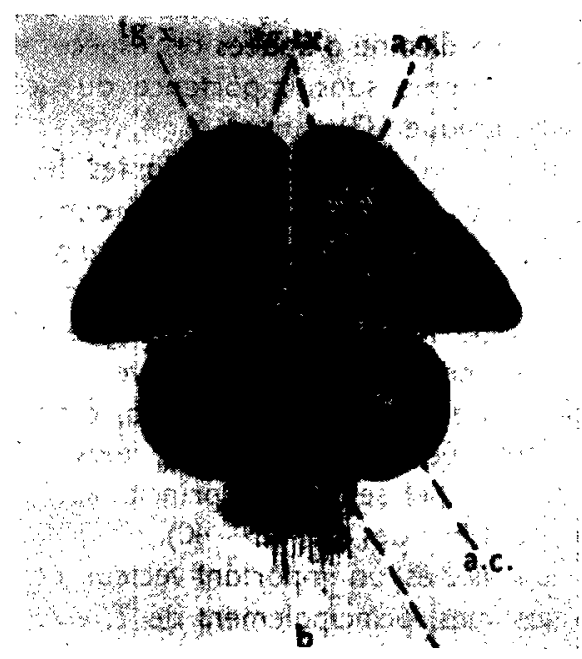

Fig. VII. — G. pallicera, femelle, plaques génitales, d'après Patton.

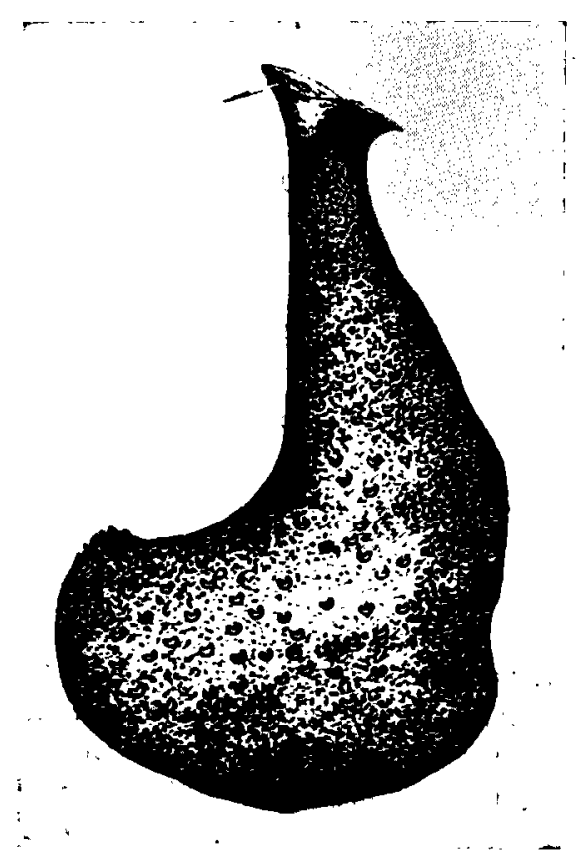

Fig. II. - G. coligineo, mâle, gonopode d'après Patton. 


\section{RÔLE VECTEUR}

Il y a une dizaine d'années ces espèces étaient réputées comme sans importance du point de vue économique (33).

En dehors des espèces courantes les chercheurs du W.A.I.T.R. * ont montré que d'autres espèces du groupe fusca et $G$. pallicera pouvaient avoir un rôle d'agent vecteur quelquefois important. En 1958, NASH (28, p. 23) souligne que cette dernière espèce est nettement un vecteur de trypanosomiase animale. En Nigeria, cette tsé-tsé a été trouvée souvent infectée, exclusivement par $T$. vivax, ef se. nourrit principalement aux dépens des bovidés $(28-29-40)$.

G. caliginea est un important vecteur de divers trypanosomes, principalement de $T$. vivax, également de $T$. congolense, rarement de trypanosomes du groupe brucei-gambiense (Cameroun 34 - 35 - 36 - 37 - 38); il n'existe que des présomptions (Nigeria - 32) que cette espèce joue un rôle dans la transmission de la maladie du sommeil à T. gambiense.

Nous n'avons aucun renseignement sur son rôle vecteur de $G$. newsteadi. L'on peut seulement présumer qu'en particulier dans certaincs régions limites de différents massifs forestiers où elle semble assez fréquente elle puisse contribuer à la diffusion de certaines trypanosomiases animales, comme on l'a observé chez $G$. pallicera en cfrique occidentale.

En Afrique centrale les espèces mentionnées ne jovent donc comme vecteur des différentes trypanosomiases qu'un rôle vraisemblablement médiocre étant donné, ou leur rareté, ou leur répartition géographique restreintc.

Institut d'élevage et de médecine vétérinaires des pays tropicaux:

Laboratoire d'entomologie (Alfort, Seine).

\section{SUMMARY}

In central Africa three species of the palpalis group, Glossina pallicera Bigot, Glossina newsteadi Austen and Glossina caliginea Austen are very scarce or strictly limited to certain areas. These species, therefore, seemed to be of very slight importance in these countries.

\section{RESUMEN}

En Africa Central tres especies del grupo palpalis: glossina pallicera Bigot, glossina newsteadi Austen y glossina caliginea Austen, tienen una distribucion muy restringuida. De acuerdo con su rareza su papel como vectores de tripanosomiasis aparece en estas regiones muy debil.

\section{BIBLIOGRAPHIE (suite) **}

30. GALLIARD $\left(\mathrm{H}_{\text {. }}\right.$. - - Glossines du Gabon occidental. Ann. Parasit. hum. comp., 1933, 11 (2) : 81-92.

31. MACHADO (A. DE BARROS), - Nouvelles contributions à l'étude systématique et biogéographique des Glossines (Diptera). Comp. Diam. Angola. Publ. Cultural, 1959, $46: 1-90$.

32. NASH (T.A.M.). - The effect of different types of man-fly confact upon the distribution of $T$. gambiense sleeping sickness in

* West African Institute for Trypanosomiasis Research Nigeria.

** Voir Maillot in Rev. Elev. Med. vet. Pays trop., 1961. 14, (4) : 439-42.
Nigeria (L'effet de différentes modalités du contact homme-tsé-tsé sur la distribution de la maladie'du sommeil à $T$. gambiense en Nigeria). C.C.T.A. Publ., 41 : 191-96.

33. PATTON (W.S.). - Studies on the higher dipter of medical and veterinary importance : a revision of the species of the genus Glossina Wiedmann based on a comparative study of the male and female genitalia ([tudes des principaux diptères d'importance médicale et vétérinaire: une révision des espèces du genre Glossina Wiedmann basée sur une étude comparative des genitalia du mâle et de la femelle). Ann. Trop. Med. Parasit., 1936, $30: 305-30$. 
34. ROUBAUD (E.). - Etude d'une infection mixte transmise à Paris par Glossina caliginea. Bull. Soc. Poth. exo., 1951, 44 : 343.

35. ROUBAUD (E.) et BOURDIE (M.). - Essais sur l'action curative et préventive de l'antrycide sur les trypanosomiases animales. Bull. Soc. Path. exo., 1950, 43 : 552-6.

36. ROUBAUD (E.) et MAILLO' (L.). - Les modalités de l'infection cyclique trypanosomienne observées chez les Glossina caliginea des gîtes à palétuviers de Douala. Bull. Soc. Path. exo., 1952, 45 (2) : 228-35.

37. ROUBAUD (E.), MAILLOT (L.) et RAGEAU (J.). - Infection naturelle de Glossina cali- ginea Austen dans les gîtes à patétuviers de Douala (Cameroun français). Bull. Soc. Path. exo., 1951, 44 (5-6) : 309-13.

38. ROUBAUD (E.), MAILLOT (L.) et RAGEAU (J.). - Infection naturelle de Glossina caliginea dans les gîtes à palétuviers de Douala (Cameroun français). Bull. Soc. Path. exo., 1952, 45 (2) : 206-8.

39. VANDERPLANK (F.L.). - Experiments in cross breeding tsetse flies. Ann. trop. Med. Parasit., 1948, 42 : 131-52.

40. West African Institute for Trypanosomiasis Research Annual, Report 1959. 